Classification

Physics Abstracts

$07.85-32.50-78.70$

\title{
A characteristic fluorescence correction factor for use in electron probe microanalysis. II. Evaluation of experimental results and comparisons
}

\author{
Christian Schiebl $\left({ }^{2}\right)$, Hans-Jürgen August $\left({ }^{\star}\right)$ and Johann Wernisch $\left({ }^{1}\right)$
}

(1) Institute für Angewandte und Technische Physik, Technische Universităt Wien, Wiedner Hauptstr. 8-10, A-1040 Wien (Vienna), Austria

(2) Digital Equipment Corp., Campus-based Engineering Center, Favoritenstr. 7, A-1040 Wien (Vienna), Austria

(Received November 29, 1990; accepted March 21, 1991)

\begin{abstract}
A comparison of a recently published secondary characteristic fluorescence correction method especially with the in electron probe microanalysis commonly used Reed procedure is presented along with evaluations of experimental data from literature taking into account the fluorescence correction using the new model. Special attention is directed to the choice of fundamental parameters, such as mass absorption coefficients, fluorescence yields, relative intensities and ionization cross sections, which are found to influence the corresponding results considerably.
\end{abstract}

\section{Introduction.}

Correction procedures in quantitative electron probe microanalysis often either neglect secondary fluorescence radiation or employ the "standard" correction procedure presented by Reed in 1965 [1]. Generally the amount of secondary radiation is small when compared to primary radiation, and therefore negligible. There are, however, cases in which characteristic secondary fluorescence contributes significantly to the total intensity and thus should be allowed for.

The present paper describes the application of a recently published [2] fluorescence correction procedure with regard to 135 experimental data taken from literature. The method takes into account Coster-Kronig transitions and effective fluorescence yields to ensure the highest possible accuracy. Moreover details of the excitation conditions, such as the subshell structures, have also been thoroughly investigated and mathematical approximations have been avoided if possible. The corresponding equation describing the secondary fluorescence correction factor $F_{j i}$, which generally is part of the so-called ZAF-correction, can be written as follows:

$$
F_{j i}=1+\frac{1}{2} \frac{A_{j}}{R_{j i}} \frac{1}{1 / S_{j i}} \frac{r_{j i}-1}{r_{j i}^{\prime}} \omega_{j i} p_{j i} \frac{1}{f_{j i}\left(\chi_{j i}\right)} \sum_{k} \frac{c_{k}}{A_{k}} \sum_{l} \frac{R_{k l}}{S_{k l}} \frac{\omega_{k l}}{\omega_{j i}} \frac{p_{k l}}{p_{j i}}\left(\frac{\tau_{k j}}{\rho_{j}}\right) f_{k l, j i}
$$

$\left.{ }^{\star}\right)$ Author to whom correspondance should be sent. 
A stands for the atomic number of the excited element, $c$ for its weight fraction, $R$ is the backscattering factor and $1 / S$ the stopping power factor. $(r-1) / r^{\prime}$ represents the absorption edge jump ratio, $\omega$ the fluorescence yield and $p$ the transition probability. Finally $f(\chi)$ and $f$ represent the absorption correction terms and $\tau / \rho$ is the photoinization cross section. The subscripts $j$ and $i$ stand for the detected radiation type $i$ of the element $j$, while $k$ and $l$ stand for all elements $k$ present in the sample, emitting various X-ray lines denoted $l$.

As can be seen from equation (1), special attention must be directed to the appropriate choice of fundamental parameters, such as the mass absorption coefficients $\mu / \rho$, fluorescence yields $\omega$, relative transition probabilities $p$, or the ionization cross section $Q$, which considerably influence the results. Supposing that the surface of the sample under investigation is flat, further improvements are not to be expected from an improvement of the theory, but from a more accurate quantification of the fundamental parameters needed for the calculation of the amount of secondary fluorescence.

In order to facilitate the interpretation of the results we have carried out our comparison mainly with regard to the most often used correction procedure following Reed [1], since this wellestablished model is found in most of the correction programs available.

\section{Effects resulting from the choice of fundamental parameters.}

Since, as given in equation (1), the fundamental parameters mainly determine the value of the fluorescence correction factor $F$ as multiplicative quantities, thus influencing the results linearly, uncertainties of these parameters may result in large errors. The choice of appropriate parameters, therefore, can be regarded as crucial with regard to the fluorescence correction procedure.

2.1 MASS ABSORPTION COEFFICIENTS $\mu / \rho$. - Although the mass absorption sets investigated by Vrebos and Pella [3] partly differ significantly from each other, it seems unjustifiable in the light of these investigations to give preference to a certain set. Sevov et al. [4], on the other hand, could show that in connection with electron probe microanalysis clear differences appear. In connection with the depth distribution functions proposed by Bastin and Heijligers [5] Sevov et al. [4] recommended the use of the values presented by Henke et al. [6] for radiation energies below $1 \mathrm{keV}$ and of those of Henrich [7] for higher radiation energies.

2.2 FLUORESCENCE YIELDS $\omega$. - In the scope of the discussed fluorescence correction procedure the subshell structure is taken into account and effective fluorescence yields are employed [2, 8]. The fluorescence yields for the K- and L-shells and the corresponding Coster-Kronig transition probabilities were taken from Krause [9]. This author presents a complete set of the parameters in question, and moreover Auerhammer et al. [10] stated that the values presented by Krause correspond better with experimental data than those of, e.g., McGuire [11] or Chen et al. [12].

Problems arise in connection with the corresponding parameters for the M-shells, since in this case unfortunately only few and uncertain values are available. While some values, though affected with considerable uncertainties, are available for the fluorescence yield [12-16], nearly no values for the Coster-Kronig transition probabilities have been published. Only the transitions from the $\mathrm{M}_{4}$ - to the $\mathrm{M}_{5}$-shell have been quantified for some elements [13]. Karttunen et al. [16] found for the elements with atomic numbers between 93 and 96 that $97 \%$ of all $\mathrm{M}_{1^{-}}, \mathrm{M}_{2-}$ and $\mathrm{M}_{3}$ vacancies pass via Coster-Kronig processes to higher subshells, viz. $\mathrm{M}_{4}$ and $\mathrm{M}_{5}$, while only the remaining 3\% of the vacancies are filled up via transitions from higher shells such as the $\mathrm{N}$ - or Oshells. This means that transitions which result in the emission of X-radiation generally end on the $\mathrm{M}_{4}$ - and $\mathrm{M}_{5}$-subshells. The mean fluorescence yield of the M-shell thus is approximately identical 
with the weighted average value of the fluorescence yields $\omega_{4}$ and $\omega_{5}$, being nearly independent of the distribution of vacancies found originally in the various subshells. Since the fluorescence yields of the $\mathrm{M}_{4}$ - and of the $\mathrm{M}_{5}$-subshell are - taking into account the margin of error - nearly identical, it can be considered as justifiable to substitute $\omega_{5}$ for the mean value of the fluorescence yield of the M-subshell structure. This will cause no large errors also because the contribution of the M-subshell to the calculation of the fluorescence correction factor $F$ is quite small.

2.3 RELATIVE INTENSITIES $p$. - For the quantification of the relative intensities $p_{\mathrm{K} \alpha}$ of $\mathrm{K}_{\alpha}$-lines a least-squares fit proposed by Poehn et al. [17], which was developed based on values published by Schreiber and Wims [18], has been employed. Due to the normalization the relative intensity $p_{\mathrm{K} \beta}$ of the $\mathrm{K}_{\beta}$-lines results in $1-p_{\mathrm{K} \alpha}$.

In case of $\mathrm{L}_{\alpha}$-lines a fit for the relative intensities $p_{\mathrm{L} \alpha}$, normalized to the sum of intensities of lines generated by transitions ending on the $\mathrm{L}_{3}$-subshell, presented by Poehn et al. [17] based on values taken from Johnson and White [19] and further developed by Schiebl [20] has been used.

For the sake of completeness also the relative intensities of the $\mathrm{M}_{\alpha}$-lines relative to the sum of intensities of lines caused by transitions ending on the $\mathrm{M}_{4}$-subshell shall be mentioned. For the quantification of these parameters a fit of Schmetterer [21] based on values of Jenkins [22] has been taken.

2.4 MEAN IONIZATION POTENTIAL $J$. - The mean ionization potential $J$ can be quantified by the formula of Zeller [unpublished, cited, e.g., in 23]:

$$
J=[10.04+8.25 \exp (-Z / 11.22)] Z / 1000
$$

According to August [24] this expression, which is similar to that presented by Ruste [25], seems to reflect the physical situation better than other quantifications found in literature. This finding is also proved by the investigations of Sevov et al. [4].

\section{Primary ionization intensities.}

Due to the normalization to the primary radiation a factor quantifying the ratio of the primary ionization intensity of the exciting element $k$ to that of the excited element $j$ is found in the fluorescence correction factor $F$ (see also Eq. (1)):

$$
\frac{l_{k l}^{p}}{l_{j i}^{p}} \propto \frac{1 / S_{k l} R_{k l}}{1 / S_{j i} R_{j i}}
$$

3.1 THE STOPPING POWER FACTOR $1 / S$. - The stopping power factor $1 / S$ is calculated from

$$
1 / S=\int_{E_{0}}^{E_{n l}} \frac{Q(E)}{\mathrm{d} E / \mathrm{d} \rho z} \mathrm{~d} E
$$

where $E_{0}$ is the electron acceleration energy, $E_{n l}$ is the edge energy, $Q(E)$ is the ionization cross section, and $\mathrm{d} E / \mathrm{d} \rho z$ quantifies the stopping power, i.e. the energy loss per unit path length.

Since the comprehensive studies carried out by August [24] have shown that the ionization cross section proposed by Pouchou and Pichoir [26] corresponds well with experimental data found for $\mathrm{K}$ - and L-shells, this approach has been implemented in the discussed model. This expression 
stands out also for the wide range of applicability with regard to the overvoltage ratio, and for the simple mathematical form.

Even more important than the dependence of the cross section on overvoltage is that the relative values of the cross sections for different shells be quantified correctly. A normalization to the maxima of the cross section curves was carried out [27] on the mathematical basis of the approach of Bethe [28] and using the Bethe-parameters proposed by Powell [29] for the K-and L-shells and by Rez [30] for the $\mathrm{M}_{4}$-shells, respectively. The resulting ionization cross section $Q_{n l}\left(U_{n l}\right), U_{n l}$ being the overvoltage ratio for the shell $n$, subshell number $l\left(U_{n l}=E / E_{n l^{\prime}} E\right.$ being the electron energy and $E_{n l}$ being the edge energy) is quantified by

$$
Q_{n l}\left(U_{n l}\right)=6.5110^{-14} \frac{z_{n l} b_{n l}}{E_{n l}^{2}} \frac{\ln \left(U_{n l}\right)}{U_{n l}^{m}}
$$

$$
\begin{aligned}
& \text { with } \quad m_{\mathrm{K}}=0.86+0.12 \exp \left(-Z^{2} / 25\right) \quad b_{\mathrm{K}}=0.5 \quad z_{\mathrm{K}}=2 \\
& m_{\mathrm{L}}=0.82 \quad b_{\mathrm{L}}=0.377 \quad z_{\mathrm{L}}=8 \\
& m_{\mathrm{M}}=0.78 \quad b_{\mathrm{M}}=0.67 \quad z_{\mathrm{M}}=18
\end{aligned}
$$

Using the stopping power $\mathrm{d} E / \mathrm{d} \rho z$ quantification of Pouchou and Pichoir [26],

$$
\mathrm{d} E / \mathrm{d} \rho z=-\frac{Z}{A J} \frac{1}{\sum_{i} D_{i}(E / J)^{P_{i}}}
$$

$$
\text { with } \begin{aligned}
D_{1}=6.6 \times 10^{-6} & P_{1}=0.78 \\
D_{2}=1.12 \times 10^{-5}\left(1.35-0.45 \mathrm{~J}^{2}\right) & P_{2}=0.1 \\
D_{3}=2.2 \times 10^{-6} / \mathrm{J} & P_{3}=0.25 \mathrm{~J}-0.5
\end{aligned}
$$

the stopping power factor $1 / S$ can be described by

$$
1 / S=z_{l} b_{l} \frac{A}{Z} \sum D_{i}\left(\frac{E_{n l}}{j}\right)^{P_{i}-1} \frac{T_{i l} U_{0}^{T_{l l}} \ln \left(U_{0}\right)-U_{0}^{T_{l l}}+1}{T_{i l}^{2}}
$$

with $U_{0}=E_{0} / E_{n l}$ and $T_{i l}=1+P_{i}-m_{l}$.

3.2 THE BACKSCATTERING FACTOR $R$. - For the calculation of the backscattering factor the expression of August et al. [31,32] has been applied, which is the result of a least-squares fit to approx. 2500 values which were calculated from fundamentals as precisely as possible.

3.3 COMPARISON OF CALCULATED INTENSITIES. - Figure 1 shows the percental deviations of the intensity ratios used in the fluorescence correction procedures according to Castaing [33], Wittry [34], Reed [1] (being originally taken from Green and Cosslett [35]), Powell [29], and Twigg and Frazer [36] compared to those calculated as described above. $\mathrm{A} \mathrm{K} \rightarrow \mathrm{K}$ excitation at various acceleration energies, viz. 20,30 and $40 \mathrm{keV}$, is assumed. The atomic number of the exciting element $Z_{k}$ is supposed to be 30 , while the atomic number of the excited element is varied.

The ratios used by Reed [1], who took the calculations of Green and Cosslett [35], show the smallest deviations, especially in cases where the secondary fluorescence is large, i.e. in cases of small atomic number differences. In these cases generally the discrepancies are smaller than 5\%.

On the other hand, extremely large deviations are found with regard to the ratios used by Castaing [33], by Twigg and Frazer [36], and, to a lesser extent, by Powell [29]. All of these quantifications yield too large ratios, while those of Wittry [34] are too small. 


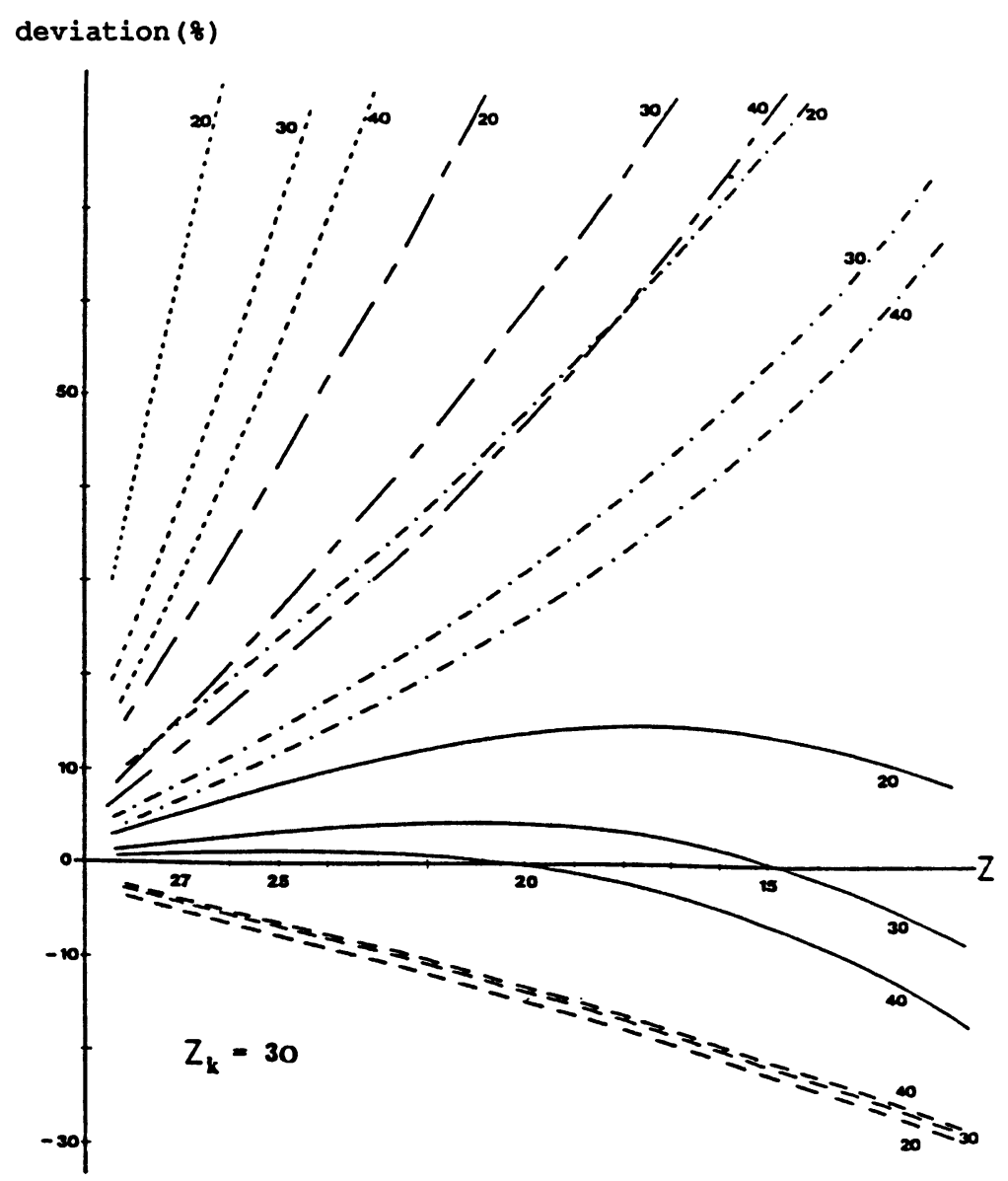

Fig. 1. - Percental deviations of the intensity ratios according to Castaing [33] (- -), Wittry [46] (--), Reed [1] (-), Twigg and Frazer [36] (- - -), and Powell [29] (- - ) compared to the values of the new model.

3.4 THE PROPORTIONALITY FACTOR $q$. - Reed [1] was the first to extent the fluorescence correction to L-radiation and he had therefore to introduce a proportionality factor $q_{k, j}$ quantifying the different ionization probabilities of the K- and the L-shells. From his own measurements he derived the values

$$
\begin{array}{ll}
q_{k, j}=1 & \text { for } k=j \\
q_{k, j}=0.24 & \text { for } k=\mathrm{K} \text { and } j=\mathrm{L} \\
q_{k, j}=4.2 & \text { for } k=\mathrm{L} \text { and } j=\mathrm{K}
\end{array}
$$

Büchner and Stienen [37] obtained

$$
q_{k, j}=\frac{\omega_{j i} p_{j i}}{\omega_{h l} p_{h l}}
$$

The index $h$ stands for a fictitious auxiliary element of which the detected $\alpha$-line should have the same radiation energy as the line of the excited element $j$, but belonging to the same shell series as the radiation of the exciting element $k$. The parameter $q_{k, j}$ thus can be calculated from values for the fluorescence yields taken from literature and relative intensities. Figure 2 shows results for a $\mathrm{K} \rightarrow \mathrm{L}$ and for a $\mathrm{L} \rightarrow \mathrm{K}$ excitation. 

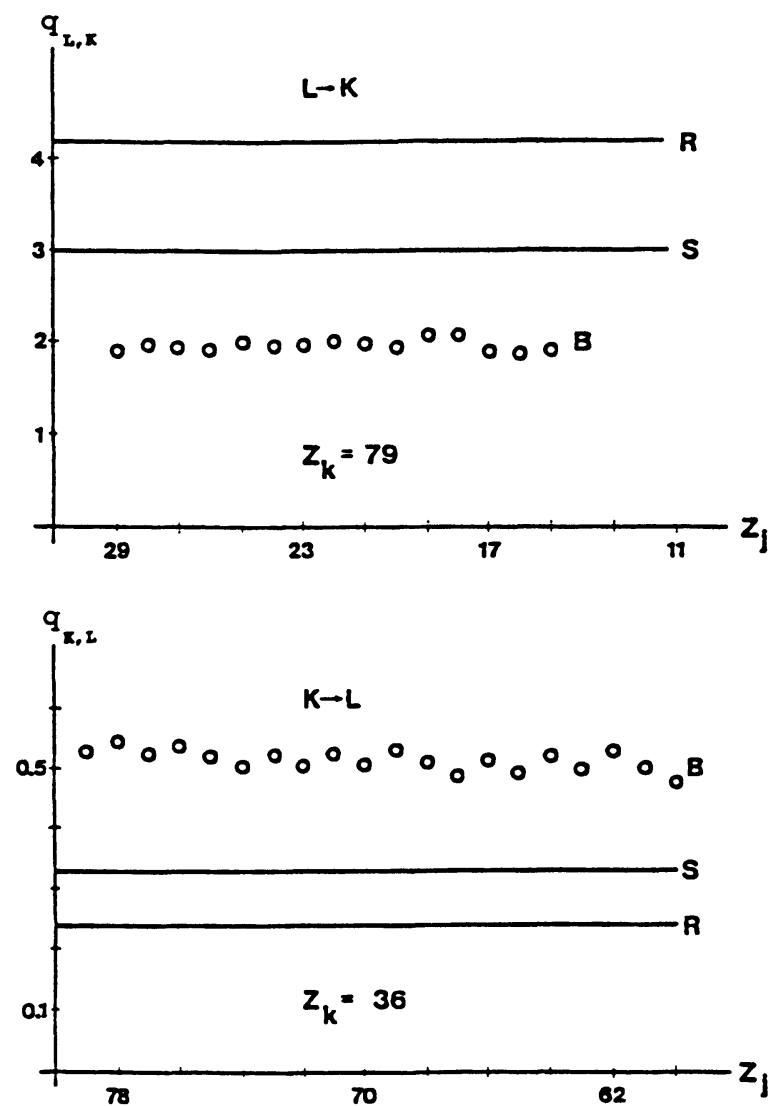

Fig. 2. - Comparison of the proportionality factors $q_{k, j}$ according to Reed [1] (R), Büchner and Stienen [37] (B) and according to the new model (S). $Z_{k}$ is the atomic number of the exciting element, $Z_{j}$ that of the excited element.

Due to the description of the primary intensities chosen in this paper the proportionality factors $q$ are given implicitely by the ratios of the Bethe-parameters discussed above, see equation (5):

$$
\begin{array}{ll}
q_{k, j}=1 & \text { for } k=j \\
q_{k, j}=1 /(8 \cdot 0.377)=1 / 3.016 & \text { for } k=\mathrm{K} \text { and } j=\mathrm{L} \\
q_{k, j}=8 \cdot 0.377=3.016 & \text { for } k=\mathrm{L} \text { and } j=\mathrm{K}
\end{array}
$$

As can be clearly seen large uncertainties are introduced in the calculation of the secondary fluorescence factor by the proportionality factor $q_{k, j}$ in case that the excited and the exciting radiation are caused by electron transitions on different shells. Since the factor $q_{k, j}$ proposed by Reed [1] was derived from few and in the meanwhile quite old measurements, and since this quantity according to Büchner and Stienen [37] is calculated from fluorescence yields and relative intensities, which again show considerable uncertainties, we suppose that the quantity presented in this paper, being based on Bethe-parameters derived directly from a large number of more recent measurements, can be considered as being the most accurate available.

Furthermore, the values given by our expression are, as can be seen from figure 2 , between those of Reed [1] and those of Büchner and Stienen [37]. 


\section{The absorption correction term.}

The value of this expression, denoted $f_{k l, j i} / f_{j i}\left(\chi_{j i}\right)$ (see Eq. (1)), is determined by the depth distribution functions of the primarily generated radiation of the exciting and of the excited element, respectively. It quantifies the ratio of the absorption corrections for the secondary fluorescence radiation to that of the primarily generated radiation of a given line of a given element, viz., the excited element.

4.1 THE DEPTH DISTRIBUTION FUNCTION $\Phi(\rho z)$. - As model for the depth distribution function $\Phi(\rho z)$ we chose to take one that is commonly used in electron probe microanalysis, viz. that of the modified Gaussian distribution. This description was first proposed by Packwood and Brown [38] and has been further elaborated by several authors [5, 39-43]:

$$
\Phi(\rho z)=[\gamma-(\gamma-\Phi(0)) \exp (-\beta \rho z)] \exp \left[-(\alpha \rho z)^{2}\right]
$$

$\Phi(0)$ is the so-called surface ionization. Generally [5, 38-41] the expression of Love et al. [44] is used for the quantification of this parameter; some recent models, though, employ new quantifications [26, 42], since the formula given by Love et al. [44] cannot be considered as being very precise $[42,45]$.

4.2 COMPARISON WITH OTHER ABSORPTION TERM QUANTIFICATIONS. - Figure 3 shows the percental deviations of the absorption terms of Wittry [46], Reed [1] (taken from Castaing [33]), Criss [47] and Heinrich [8], respectively, from those calculated in the present paper for a $K \rightarrow K$ excitation. The exciting element is nickel, and the excited elements have atomic numbers ranging from $Z=18$ to $Z=26$.

The differences appearing result from the use of different primary radiation distributions. Reed [1] took the expression suggested by Castaing [33], assuming the depth distribution to be an exponential function. The same model was also used by Criss [47] and by Heinrich [8], although these authors took into account the $f_{k l}\left(\chi_{j i}\right) / f_{j i}\left(\chi_{j i}\right)$ ratio, which usually is neglected. Wittry [46] dispensed with a depth distribution and assumed a point on the sample's surface to be the only source of generated primary radiation.

As can be clearly seen from figure 3 , the results according to Wittry [46] show the largest deviations; this could be expected in the light of the above mentioned simplifications used in his model. The expressions of Reed [1] and Castaing [33] on the one hand and of Criss [47] and Heinrich [8] on the other hand yield similar values. This is not surprising, since the value of the $f_{k l}\left(\chi_{j i}\right) / f_{j i}\left(\chi_{j i}\right)$ ratio does not deviate strongly from unity, since the corresponding depth distribution functions are similar. This also explains why the deviations increase with increasing atomic number difference between the exciting and the excited element, since the similarity of the corresponding depth distribution functions and of the mass absorption coefficients becomes smaller with larger atomic number differences.

Compared to the values calculated using realistic depth distributions those obtained from the expression of Reed [1] and Castaing [33] show only small deviations in cases where the secondary fluorescence is large, i.e. in cases of small atomic number differences. The errors are generally smaller than 5\%, which means that the introduction of a simplified exponential depth distribution function for the primary radiation does not significantly affect the results. 


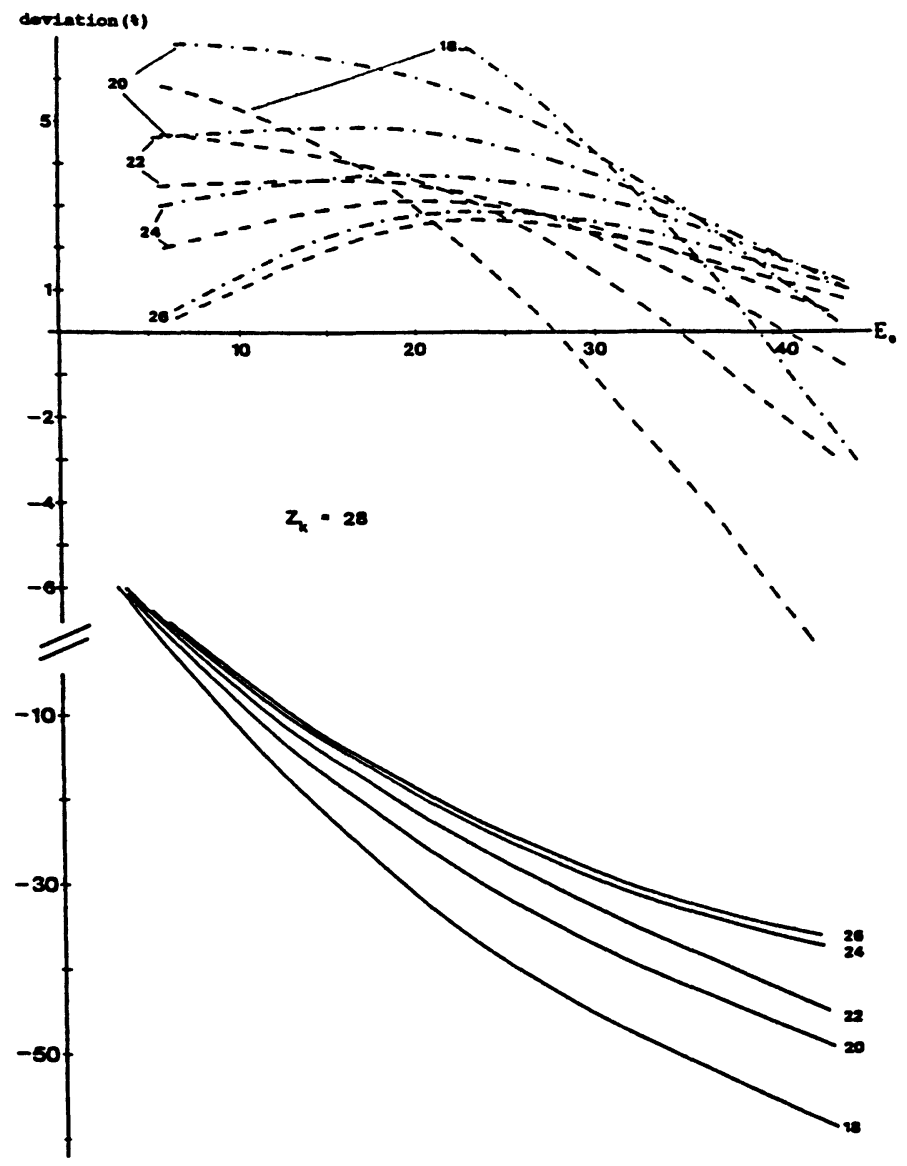

Fig. 3. - Percental deviations of the absorption terms according to Reed [1] and Castaing [33] (--), to Criss [47] and Heinrich [8] $(\cdots)$, and to Wittry [46] (-) compared to the values of the new model.

\section{Comparison of secondary fluorescence correction factors.}

Figures 4-7 show comparisons of various fluorescence correction factors. The correction factors were of course calculated using the same fundamental parameters so as to illustrate the effects caused by the different calculations.

The deviations are generally smallest in case of $\mathrm{K} \rightarrow \mathrm{K}$ excitations (Fig. 4), which can partly be attributed to the fact that this type of excitation has been investigated quite frequently. It is interesting that the ratios obtained from Wittry's more sophisticated formula, which takes into account the depth distributions of primary radiation [34], shows larger deviations than those according to Wittry's simplified model [46] assuming a point source on the sample's surface. The values calculated by means of the new formula are especially similar to those obtained from Reed's [1] expression. Similar results are also obtained using the models of Ugarte et al. [48], viz., either that assuming the depth distribution function to be quantifiable by a sum of three exponential functions - following the proposal of Criss and Birks [49] - (Ugarte 1), or that substituting a delta function for the real distribution (Ugarte 2). 


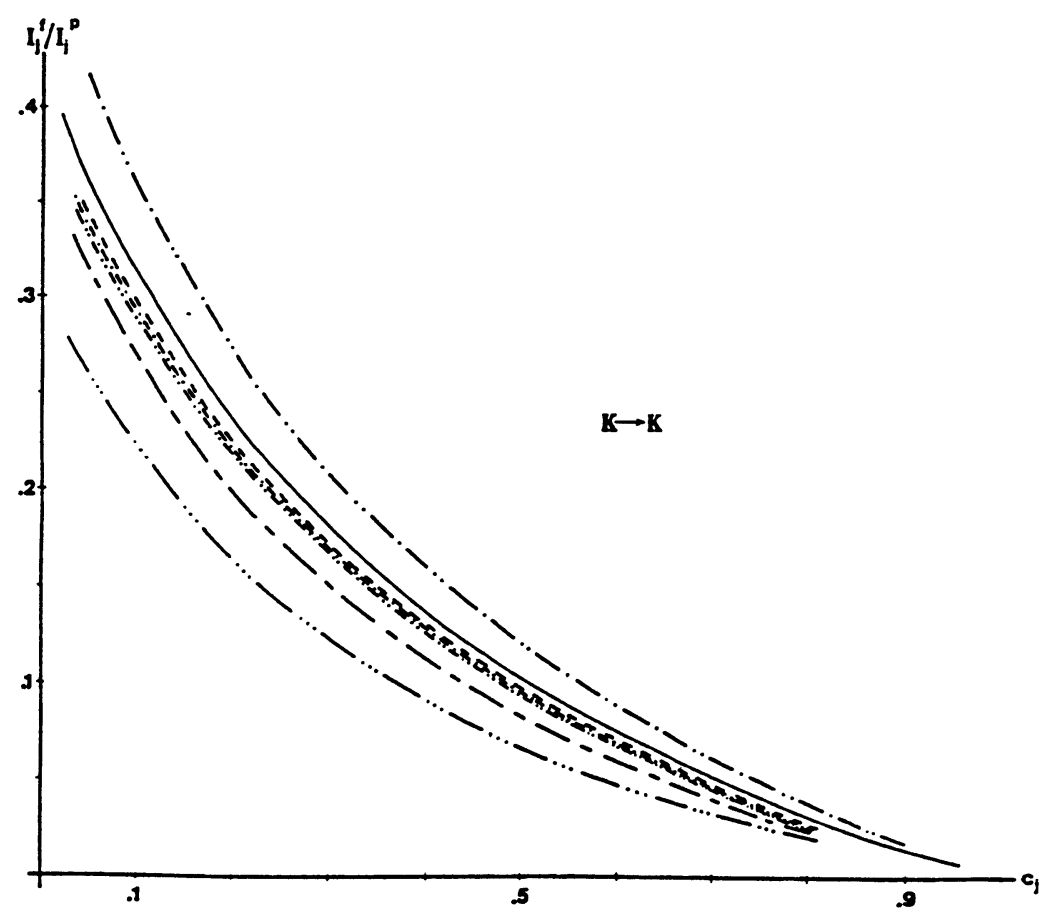

Fig. 4. - The ratio of secondary to primary radiation $l_{j}^{f} / l_{j}^{p}$ for a $\mathrm{K} \rightarrow \mathrm{K}$ excitation. Fe excites $\mathrm{Cr}, E_{0}=$ $30 \mathrm{keV}$, values as a function of the $\mathrm{Cr}$ concentration. Models: Reed [1] (- - ), Wittry $1[46](---)$, Wittry 2

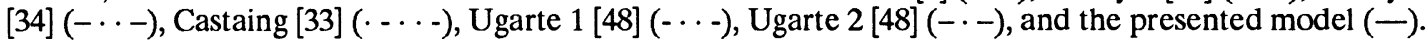

Significantly different results are obtained in the cases of excitations involving the L-shells. Figure 5 illustrates the situation for a $\mathrm{L} \rightarrow \mathrm{L}$ excitation. The new values are considerably larger than those of all other authors, but closest to those of Castaing [33]. The largest discrepancies are between our values and those of the delta-function model of Ugarte et al. [48], denoted Ugarte 2.

The differences for a $\mathrm{K} \rightarrow \mathrm{L}$ secondary fluorescence excitation are slightly smaller. Again the new values are the highest, and again those of Castaing [33] are the closest. Strong deviations are found for both models of Ugarte et al. [48] and especially for that of Reed [1].

The last set of comparative curves relates to the case of a $\mathrm{L} \rightarrow \mathrm{K}$ process, yielding the largest discrepancies. The new values are considerably lower than the others, being closest this time to those of Reed [1]. The most dramatic difference with our values is with the expression of Castaing [33], the latter values being more than $120 \%$ higher than those calculated using our model.

These differences can mostly be attributed to the different calculation of the primary intensities of K- and of L-radiation, the ratio of which determines the proportionality factor $q$, which has already been discussed above.

\section{Evaluation of experimental data.}

For the purpose of a test of the new secondary fluorescence correction model 135 experimental values have been extracted from a database of 554 measurement data sets presented by Sewell $e t$ al. [50]. To illustrate the effects caused by the choice of different depth distribution functions both the absorption correction and the fluorescence correction are calculated using a variety of depth 


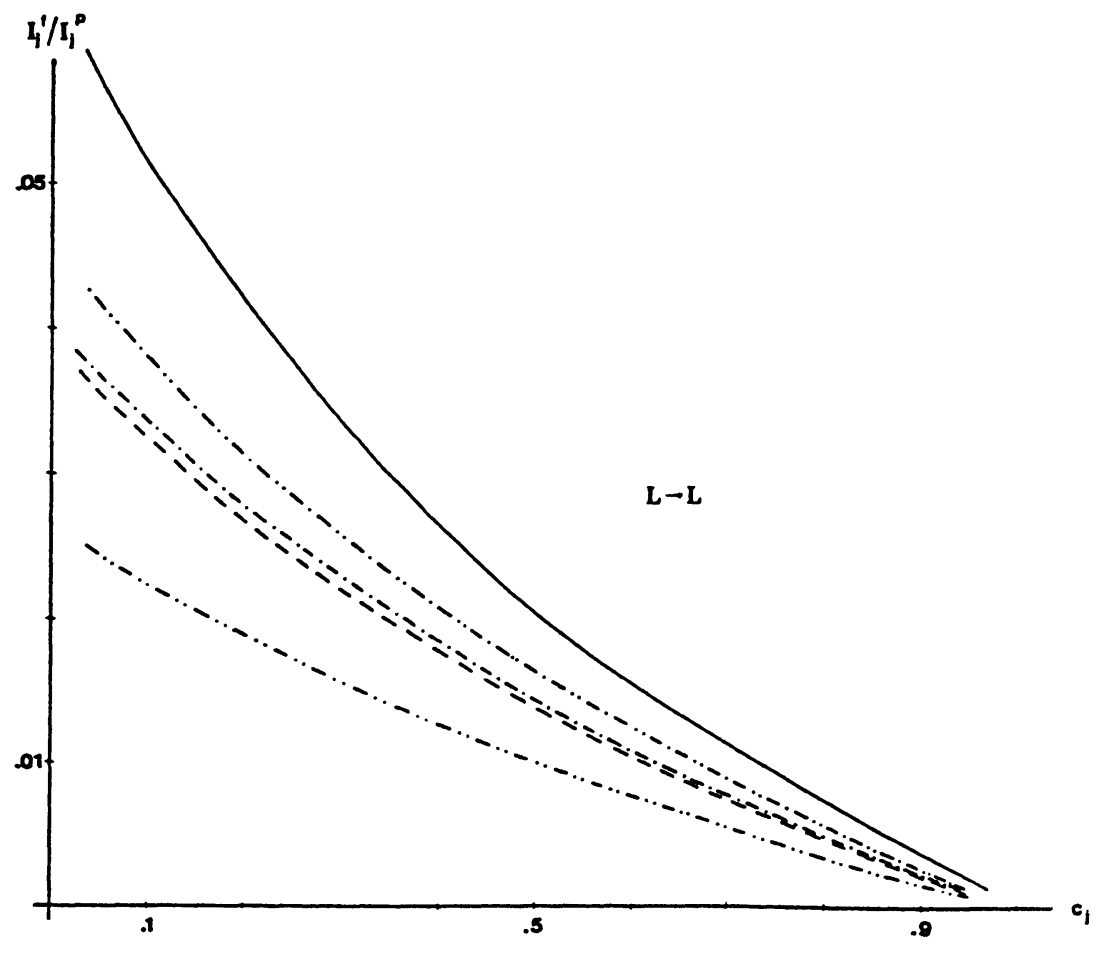

Fig. 5. - The ratio of secondary to primary radiation $I_{j}^{f} / I_{J}^{p}$ for a $\mathrm{L} \rightarrow \mathrm{L}$ excitation. Sn excites $\mathrm{Ag}, E_{0}=$ $25 \mathrm{keV}$, values as a function of the $\mathrm{Ag}$ concentration. Models: Reed [1] (- - ), Castaing [33] $(\cdots-)$, Ugarte $1[48](-\cdots)$, Ugarte $2[48](-\cdots)$, and the presented model (-).

distribution models, viz., that of Packwood and Brown [38], that of Bastin et al. published in 1984 $[39,40]$, that of Bastin et al. published two years later [41]; that of Bastin and Heijligers [5], and finally that of Rehbach and Karduck [42].

The characteristic fluorescence is calculated on the one hand using the approach of Reed [1] being the most common one, and on the other hand employing our model.

All of the depth distibutions mentioned above in principle allow a combined calculation of both the atomic number correction factor $\mathbf{Z}$ and the absorption correction factor $\mathbf{A}$. It is, however, possible to separate these correction factors and to calculate the atomic number correction factor $\mathbf{Z}$ explicitely. A first investigation, therefore, concerned the effect of this separate calculation of these factors on the evaluation of the experimental data. For this purpose we applied the stopping power factor $1 / S$ according to Pouchou and Pichoir [26] and the backscattering factor following August et al. [31, 32]. The results were quite remarkable, since the separate calculation gave significantly better results, reducing the root mean square error of the ratio $k^{\prime} / k$ of calculated to measured $k$-ratios by more than $1 \%$, except in the case of the depth distribution of Packwood and Brown [38], where no significant alterations could be found. As a result of these investigations we chose to use this mode of calculation of the $\mathbf{Z}$-and $\mathbf{A}$-correction factors for the evaluation of the experimental data.

The comparisons concerning the various models of depth distribution functions are depicted in figures 8-12. As can be clearly seen, the root mean square error resulting from the new calculations is always smaller than that obtained when using Reed's [1] expression. On the other hand, the 


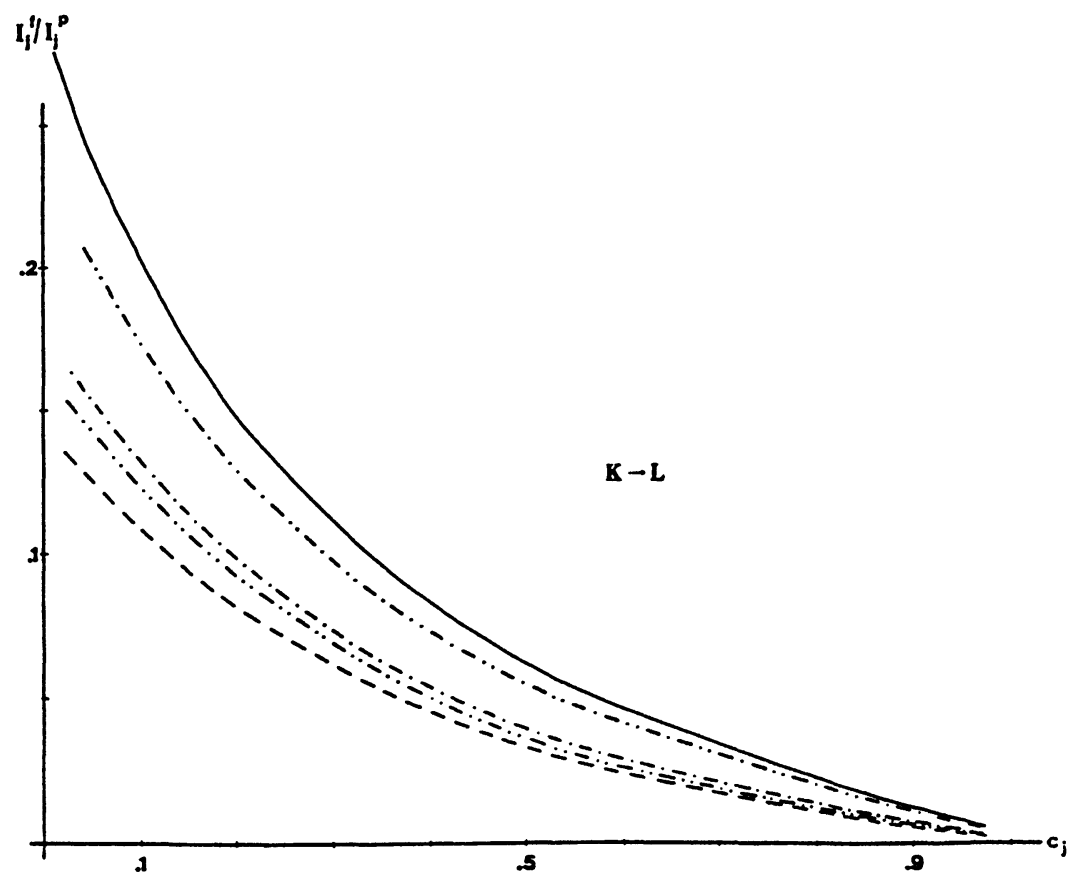

Fig. 6. - The ratio of secondary to primary radiation $I_{j}^{f} / I_{J}^{p}$ for a $\mathrm{K} \rightarrow \mathrm{L}$ excitation. Rb excites $\mathrm{Au}$, $E_{0}=25 \mathrm{keV}$, values as a function of the Au concentration. Models: Reed [1] (- - ), Castaing [33] (. - .. -), Ugarte $1[48]$ (- .. -), Ugarte $2[48]$ (- . -), and the presented model (-).

average value according to Reed is always somewhat higher than ours, which generally means that it is closer to unity, except in the case of the depth distribution funtion of Rehbach and Karduck [42].

To facilitate a further analysis of these results the measurements are subdivided in sets characterized by the type of excitation, viz. $\mathrm{K} \rightarrow \mathrm{K}, \mathrm{L} \rightarrow \mathrm{L}, \mathrm{K} \rightarrow \mathrm{L}$, or $\mathrm{L} \rightarrow \mathrm{K}$. Unfortunately the amount of experimental data involving $\mathrm{K} \rightarrow \mathrm{K}$ or $\mathrm{L} \rightarrow \mathrm{L}$ excitations is very small (15 and 21 measurements, respectively), so that our investigations are restricted to the cases of $\mathrm{K} \rightarrow \mathrm{L}$ and $\mathrm{L} \rightarrow \mathrm{K}$ excitations (56 and 43 measurements, respectively). On the other hand this restriction does not result in a considerable loss of information, because the correction procedure presented here shows results similar to those of Reed [1] in case of $\mathrm{K} \rightarrow \mathrm{K}$ or $\mathrm{L} \rightarrow \mathrm{L}$ excitations. Since the use of the depth distribution function of Bastin and Heijligers [5] gave the best results, see figure 11, this quantification of the distribution of primary X-rays in depth was chosen for the calculation of the values shown in the following.

Figures 13 and 14 show the results obtained for $\mathrm{K} \rightarrow \mathrm{L}$ and for $\mathrm{L} \rightarrow \mathrm{K}$ excitations, respectively. In both cases the root mean square error is smaller for the new fluorescence correction model. This characteristic already appeared in figures 8-12, where no separation of the excitation type was made. However, the behaviour of the mean value now differs: In case of $\mathrm{K} \rightarrow \mathrm{L}$ excitation it is too low for both models, although the mean value obtained for the new model is closer to unity. The situation in case of $\mathrm{L} \rightarrow \mathrm{K}$ excitation shows higher mean values for both models, that for the new one being still slightly below unity, while that resulting from the expression of Reed [1] is larger than unity. It is this characteristic behaviour of the mean values for $k^{\prime} / k$ of Reed's model which, in sum, leads to the quite good mean values appearing in figures 8-12. Although not shown 


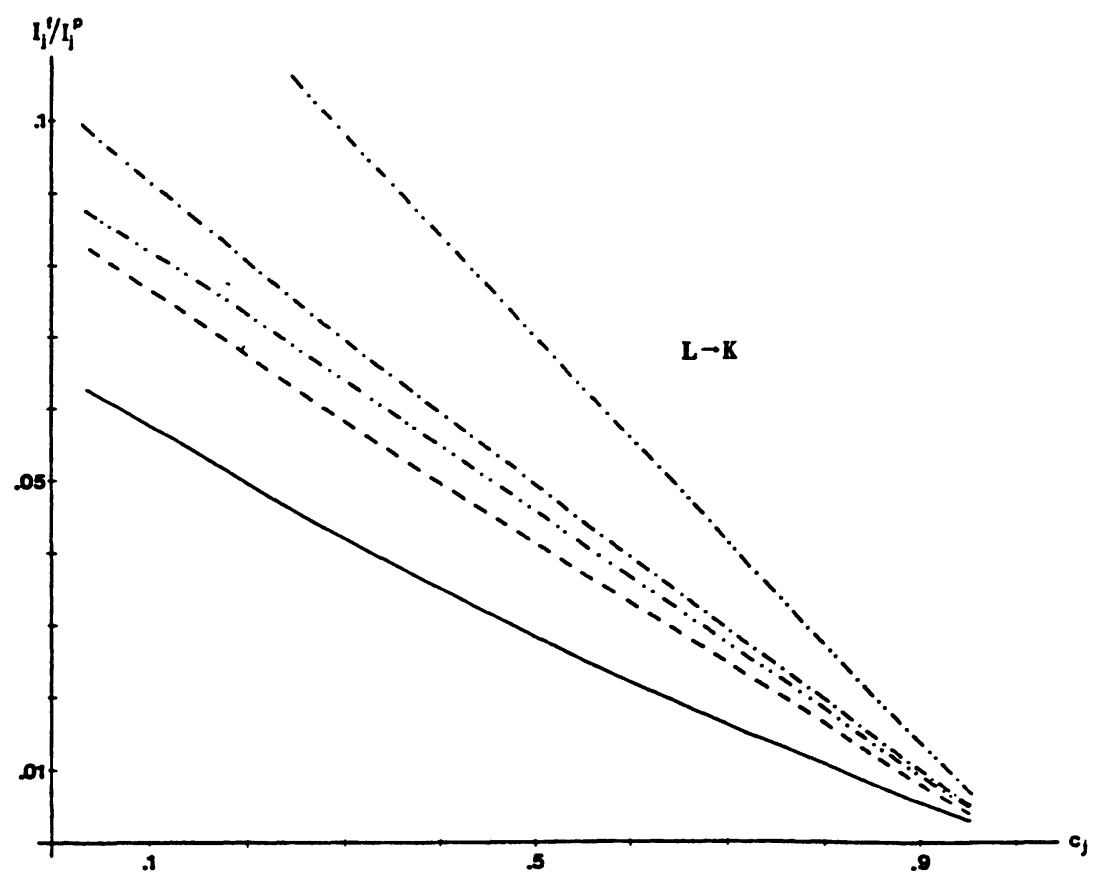

Fig. 7. - The ratio of secondary to primary radiation $I_{j}^{f} / I_{J}^{p}$ for a $\mathrm{L} \rightarrow \mathrm{K}$ excitation. Au excites $\mathrm{Cu}, E_{0}=$ $25 \mathrm{keV}$, values as a function of the Au concentration. Models: Reed [1] (---), Castaing [33] ( - . - -), Ugarte $1[48](-\cdots)$, Ugarte $2[48](--)$, and the presented model $(-)$.

explicitely, it should be mentioned that in the cases of $\mathrm{K} \rightarrow \mathrm{K}$ and of $\mathrm{L} \rightarrow \mathrm{L}$ excitation the situation is very similar to that found for $\mathrm{K} \rightarrow \mathrm{L}$ excitation.

Figure 15 shows the results obtained from the evaluation of data where the secondary fluorescence intensity exceeds $1 \%$ of the total radiation intensity (58 measurements). Again a considerable improvement of the root mean square error appears when using the new expression, although the mean value for $k^{\prime} / k$ is worse.

It is not clear whether this systematic underestimation of the $k$-ratio is due to the secondary fluorescence correction or to the other corrections employed in the calculation, although it is quite interesting that the mean value for $k^{\prime} / k$ obtained increases when more recent depth distribution models are applied, as can be seen from figures 8-12. Moreover, since the extent of the fluorescence correction is small compared to that of the other corrections, viz. the atomic number and the absorption corrections, the latter are likely to be responsible for the systematic deviations. Of course also the errors introduced by the fundamental parameters must be considered; Bastin and Heijligers [51] and Sevov et al. [4] showed remarkable changes of the $k^{\prime} / k$-values resulting from even small uncertainties of the mass absorption coefficients. 

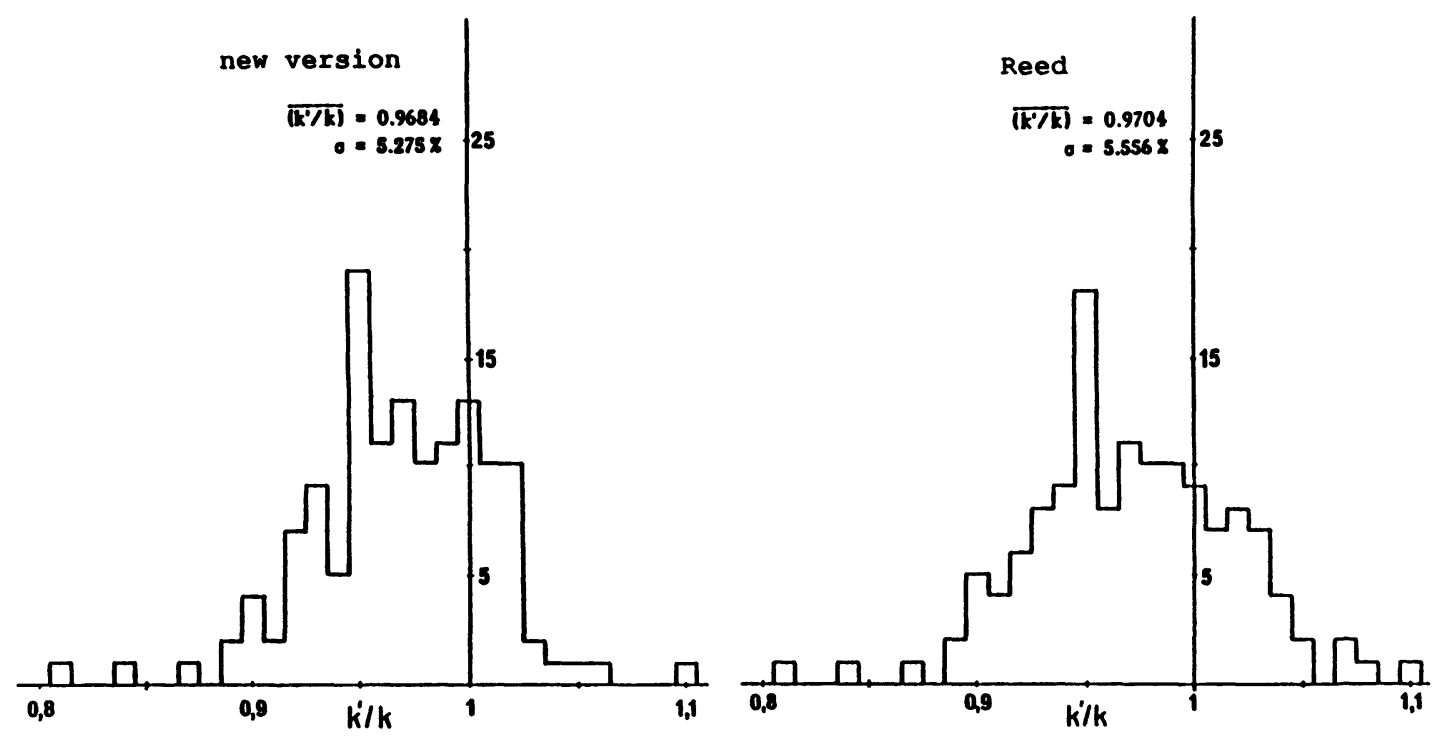

Fig. 8. - Histograms showing the distribution of $k^{\prime} / k$-ratios for 135 data sets. Left: the new secondary fluorescence correction, right: the model of Reed [1]. Depth distribution function according to Bastin and Brown [38].
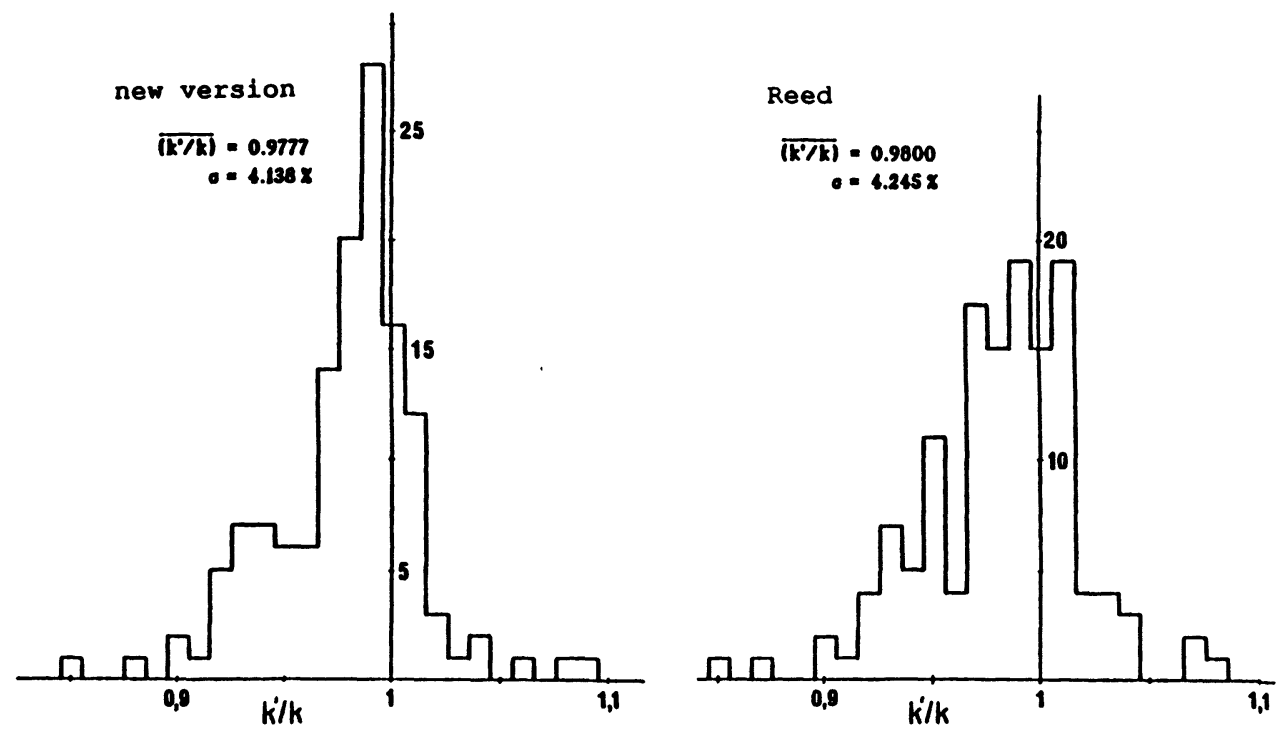

Fig. 9. - Histograms showing the distribution of $k^{\prime} / k$-ratios for 135 data sets. Left: the new secondary fluorescence correction, right: the model of Reed [1]. Depth distribution function according to Bastin et al. $[39,40]$. 

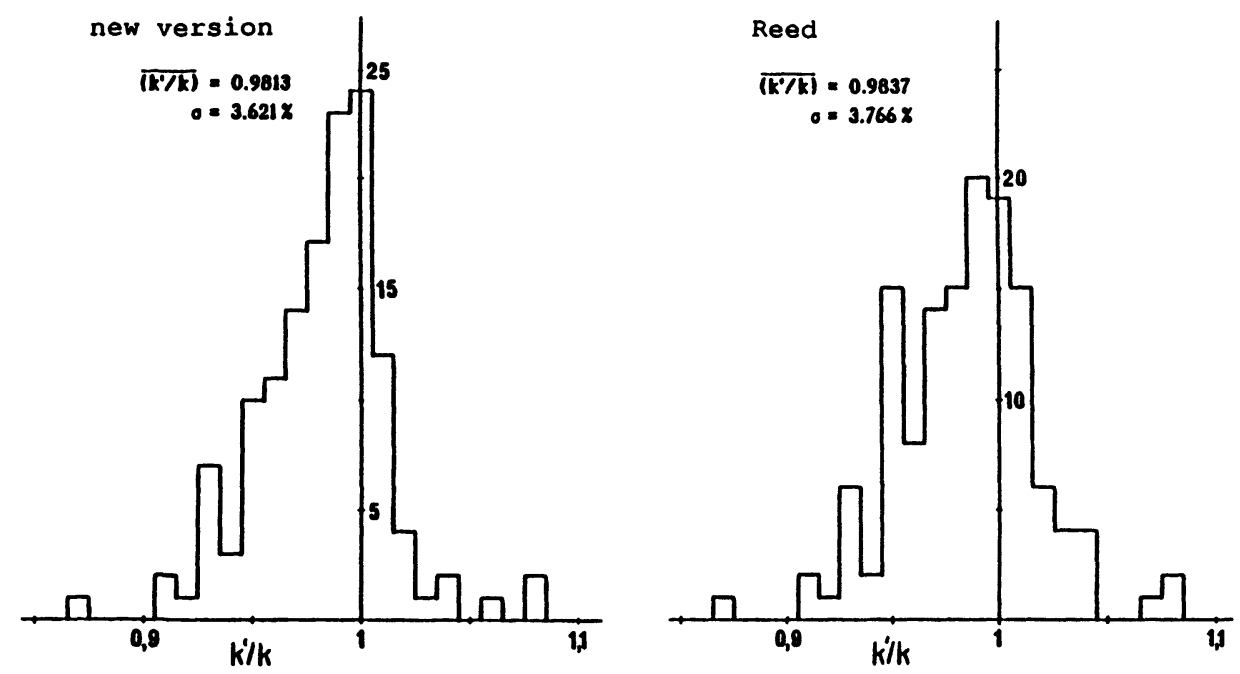

Fig. 10. - Histograms showing the distribution of $k^{\prime} / k$-ratios for 135 data sets. Left: the new secondary fluorescence correction, right: the model of Reed [1]. Depth distribution function according to Bastin et al. [41].
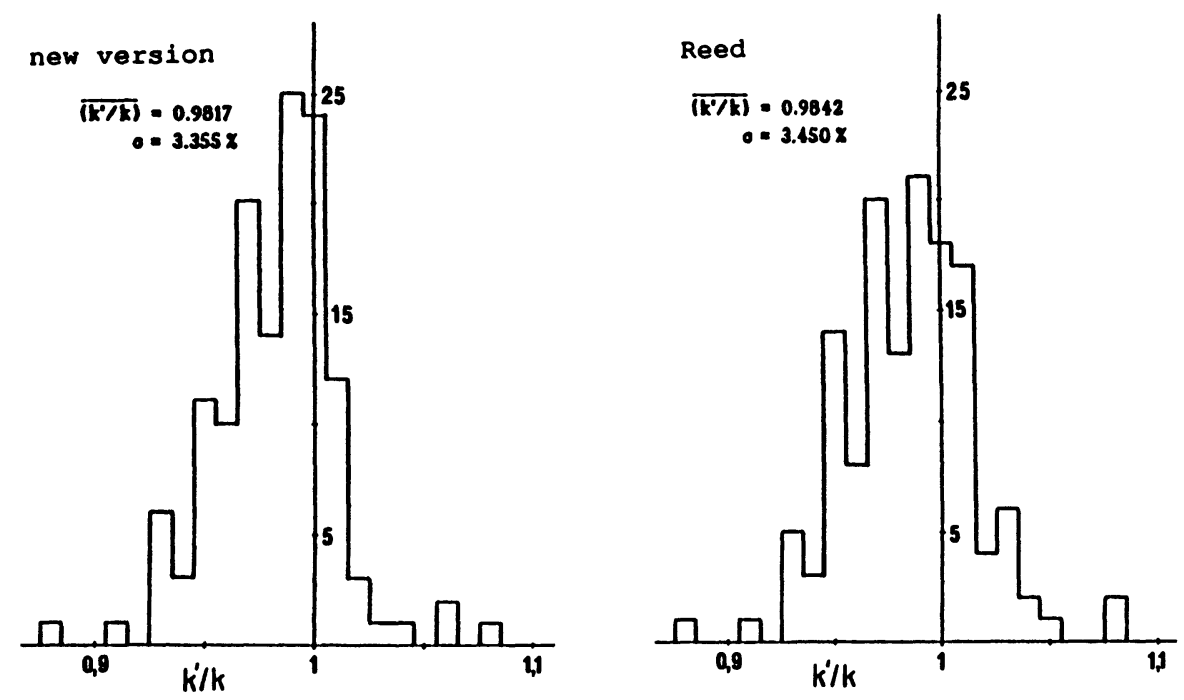

Fig. 11. - Histograms showing the distribution of $k^{\prime} / k$-ratios for 135 data sets. Left: the new secondary fluorescence correction, right: the model of Reed [1]. Depth distribtuion function according to Bastin and Heijligers [5].

\section{Conclusions.}

The recently published [2] expression for the secondary fluorescence correction factor has been thoroughly tested, applying it to the evaluation of experimental data taken from literature. Various comparisons with other fluorescence corrections, especially with that presented by Reed, 

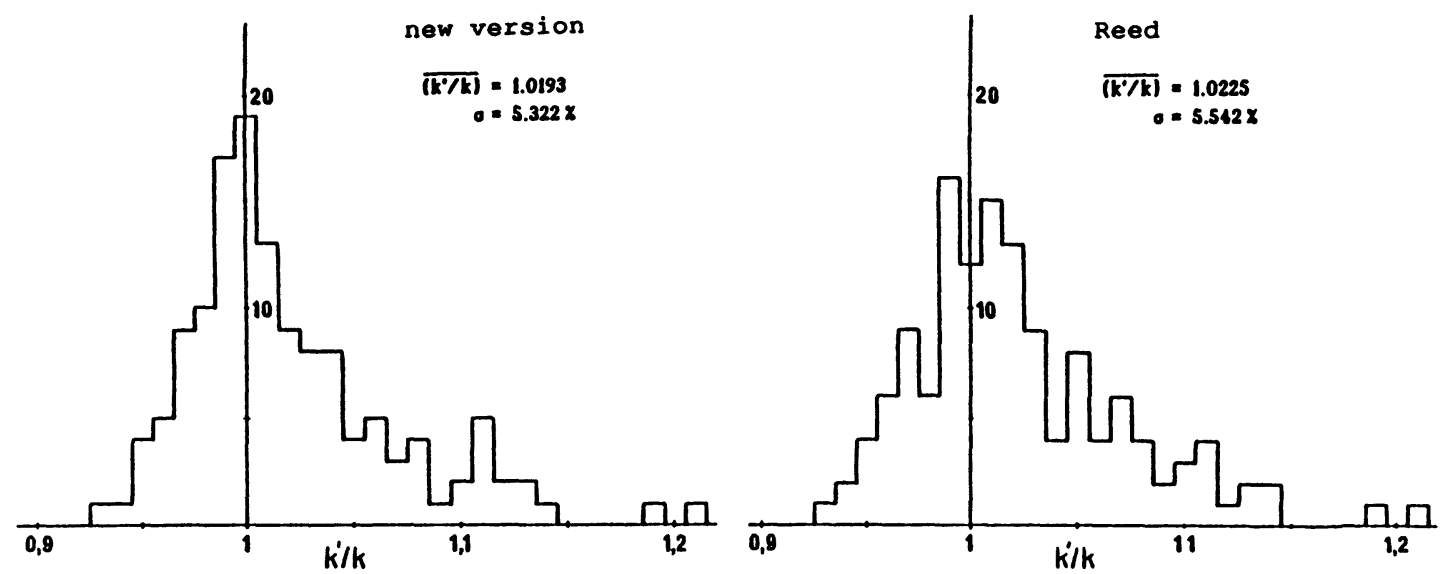

Fig. 12. - Histograms showing the distribution of $k^{\prime} / k$-ratios for 135 data sets. Left: the new secondary fluorescence correction, right: the model of Reed [1]. Depth distribution function according to Rehbach and Karduck [42].
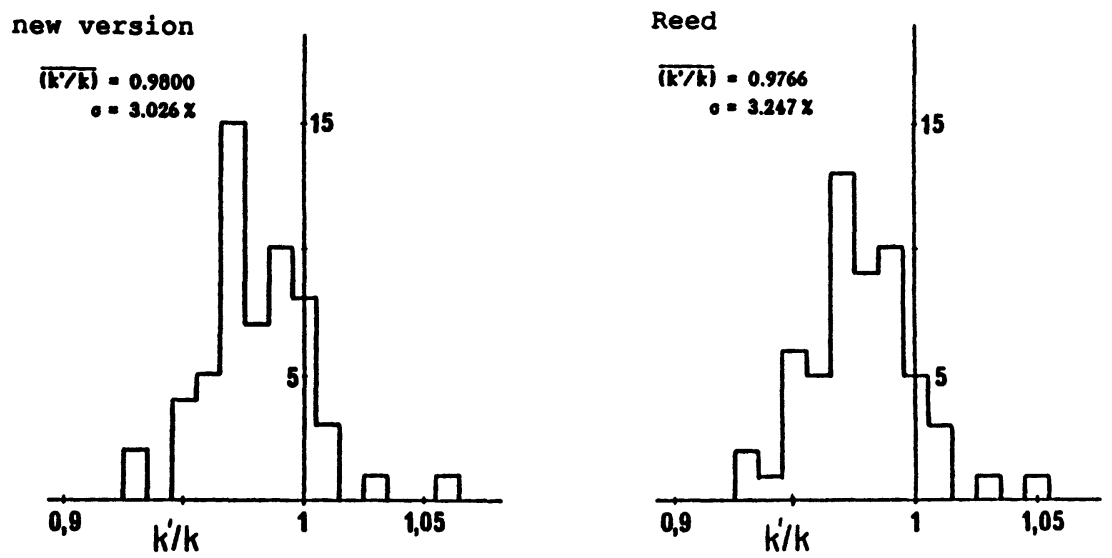

Fig. 13. - Histograms showing the distribution of $k^{\prime} / k$-ratios for $\mathrm{K} \rightarrow \mathrm{L}$ excitation (56 data sets). Left: the new secondary fluorescence correction, right: the model of Reed [1]. Depth distribtuion function according to Bastin and Heijligers [5].

which is still commonly used, have been carried out to illustrate the characteristics of these different models. The evaluation of 135 measurements shows that the new model leads to better root mean square errors than that of Reed, while the mean ratios of calculated to measured values of the latter are closer to unity. This phenomenon, however, is due to the too large mean values in case of $\mathrm{L} \rightarrow \mathrm{K}$ excitation, which compensate the errors resulting from the too low values found in cases of other excitation processes, leading to an overall value close to unity.

Considerable uncertainties are introduced through the necessary fundamental parameters such as the mass absorption coefficients $\mu / \rho$, the photoionization coefficients $\tau / \rho$, the fluorescence yields $\omega$, and the transition probabilities $p$. Most of these parameters influence the results linearly, and consequently errors and uncertainties are transferred directly to the secondary fluorescence factor. 

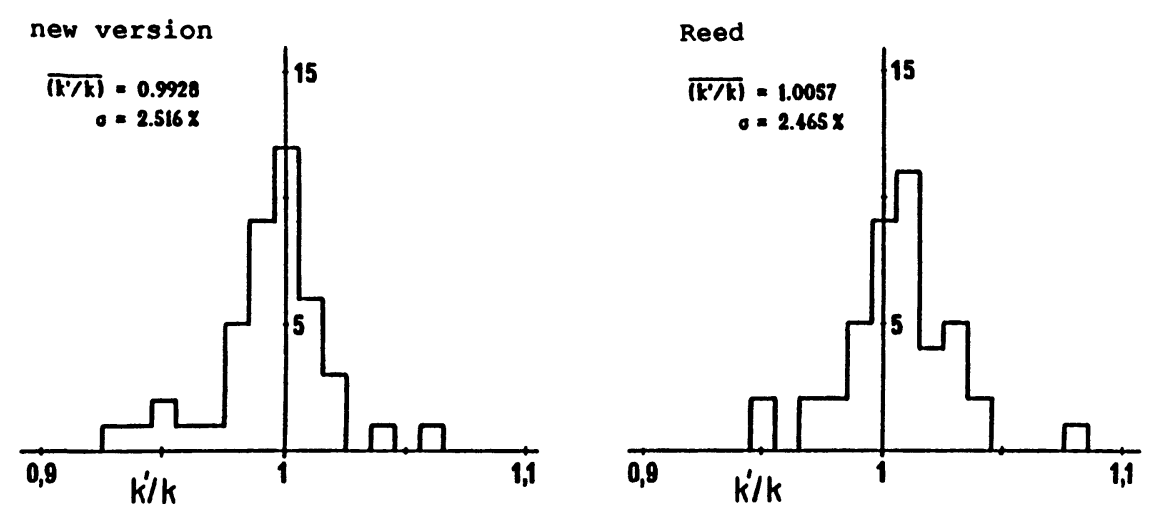

Fig. 14. - Histograms showing the distribution of $k^{\prime} / k$-ratios for $\mathrm{L} \rightarrow \mathrm{K}$ excitation (43 data sets). Left: the new secondary fluorescence correction, right: the model of Reed [1]. Depth distribtuion function according to Bastin and Heijligers [5].
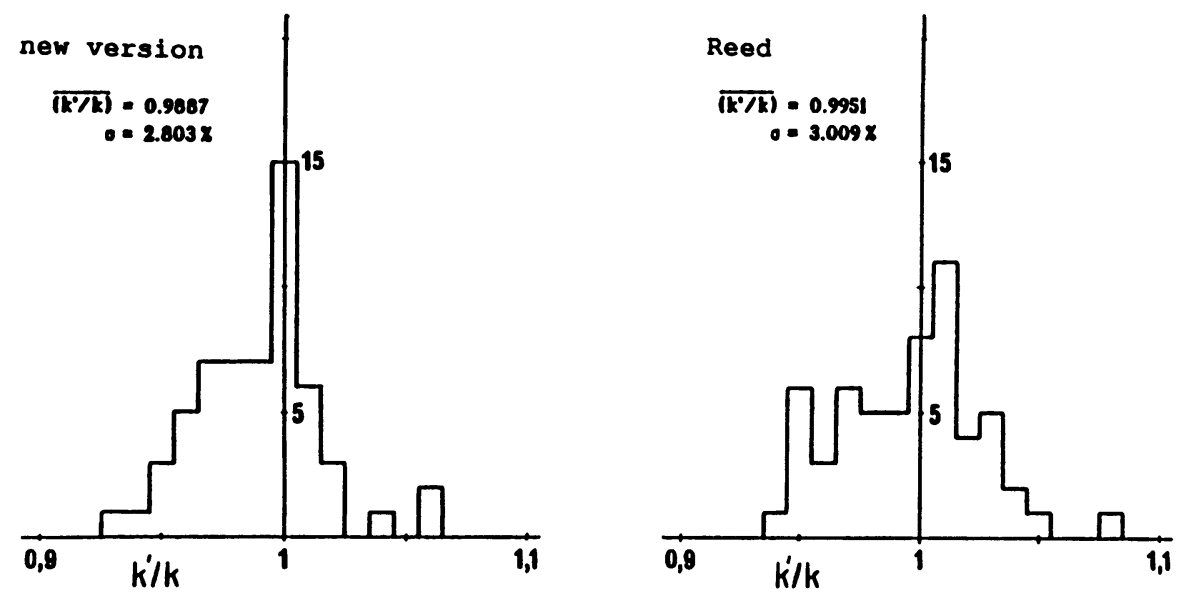

Fig. 15. - Histograms showing the distribution of $k^{\prime} / k$-ratios for cases in which the intensity of secondary fluorescence radiation exceeds $1 \%$ of the total intensity ( 58 data sets). Left: the new secondary fluorescence correction, right: the model of Reed [1]. Depth distribtuion function according to Bastin and Heijligers [5].

The proportionality factor $q$, which describes the different efficiencies appearing at the ionization of different shells, also represents a source of uncertainty, if the exciting and the excited radiation originate from different shells, i.e. in case of $\mathrm{K} \rightarrow \mathrm{L}$ or of $\mathrm{L} \rightarrow \mathrm{K}$ excitations. The corresponding uncertainties are also illustrated by the considerable differences connected with the quantification of this factor according to different authors.

Furthermore it is shown that the choice of depth distribution function does not influence considerably the results. The deviations caused by unrealistic depth distributions, e.g. the exponential one proposed by Reed, are generally smaller than $5 \%$.

Further improvements of the secondary fluorescence correction factor for characteristic radiation, therefore, are mainly not to be expected from a further refinement of the basic theory, but from a more accurate quantification of the fundamental parameters, especially of the mass 
absorption coefficient $\mu / \rho$ and of the fluorescence yield $\omega$, and from better descriptions of the absolute values of the ionization cross sections, since these determine the value of the proportionality factor $q$ discussed above.

\section{Acknowledgements.}

The authors acknowledge the support given by the Austrian Fonds zur Förderung der wissenschaftlichen Forschung (Projekt P7336 Phy).

\section{References}

[1] ReED S. J. B., Brit. J Appl. Phys. 16 (1965) 913.

[2] SCHIEBL C., AUGUST H.-J. and WERNISCH J. this volume.

[3] VRebos B. A. R. and Pella P. A. X-Ray Spectrom. 17 (1988) 3.

[4] Sevov S., Degischer H. P., August H.-J. and WernisCh J., Scanning 11 (1989) 123.

[5] Bastin G. F. and HeiJligers H. J. M., Quantitative Electron Probe Microanalysis of Boron in Binary Borides. Report of the University of Technology Eindhoven, Laboratory for Physical Chemistry, PO Box 513, 5600 MB Eindhoven, ISBN 90-6819- 006-7 CIP, 1986.

[6] Henke B. L., Lee P., TanaKa T. J., Shimabukuro R. L. and Fujikawa B. K., Atom. Data Nucl. Tables 27 (1982) 1.

[7] HeINRICH K. F. J., in: 11th Int. Cong. on X-Ray Optics and Microanal. (London, Ontario, 1986). J. D. Brown and R. H. Packwood Eds. London/Ontario (1986) 67.

[8] HeINRICH K. F. J. Electron Beam X-Ray Microanalysis (van Nostrand Reinhold Co., New York, 1981).

[9] Krause M. O., J. Phys. Chem. Rev. Data 8 (1979) 307.

[10] AUERHAMMER J., GENZ H. and RiCHTER A., Z. Phys. D 7 (1988) 301.

[11] Mcguire E. J., Phys. Rev. A 3 (1971) 587.

[12] Chen M. H., Craseman B. and MaRK H. Phys. Rev. 21 (1981) 449.

[13] Mcguire E. J., Phys. Rev. A 5 (1972) 1043.

[14] Konstantinov A. A. and SaZOnOva T. E. Izv. Akad. Nauk. SSSR, Ser. Fiz. 32 (1968) 631.

[15] Jopson R. C., Mark H., Swift C. D. and Williamson M. A., Phys. Rev. 137 A (1965) 1353.

[16] KartTunen E., Freund H. U. and Fink R. W. Phys. Rev. A 4 (1971) 1995.

[17] POEHN C., WERNISCH J. and HANKE W., X-Ray Spectrom. 14 (1985) 3.

[18] SCHREIBER T. P. and WIMS A., X-Ray Spectrom. 11 (1982) 42.

[19] JoHnson Jr. G. G. and WHITE W., ASTM Data Series DS 46 (American Society for Testing and Materials, Philadelphia, Pa., 1970).

[20] SCHIEBL C., Thesis (Technische Universităt Wien, Vienna, 1987).

[21] SCHMETTERER L., Thesis (Technische Universität Wien, Vienna, 1986).

[22] JENKINS R., X-Ray Spectrom. 2 (1973) 207.

[23] Ruste J. and Gantois M., J. Phys. D: Appl. Phys. 8 (1975) 872.

[24] AuguST H.-J. Doctoral Thesis (Technische Universităt Wien, Vienna, 1988).

[25] RUSTE J., J. Microsc. Spectrosc. Electron. 4 (1979) 123.

[26] PouchOU J.-L. and PICHOIR F., in: 11th Int. Cong. on X-Ray Optics and Microanalysis (London, Ontario, 1986). Brown J. D. and Packwood r. h. Eds. London/ Ontario (1986) 247.

[27] SCHIEBL C., Doctoral Thesis, (Technische Universităt Wien, Vienna, 1989).

[28] Bethe H. A., Ann. Phys. 5 (1930) 325.

[29] POWELl C. J., in: NBS Spec. Publ. 460 (1976) 97.

[30] REZ P., X-Ray Spectrom. 13 (1984) 55.

[31] AuguST H.-J., RAZKA R. and WERNISCH J., Scanning 10 (1988) 107.

[32] August H.-J. and WERNisCH J., Radex-Rundschau 1988-2/3 (1988) 624.

[33] Castaing R., Ph. D. Thesis, University of Paris (ONERA Publ. Nr.), (1951) 55.

[34] WITTRY D. B., University of Southern California Engeneering Center, Report 84-204, 1962.

[35] Green M. and Cosslett V. E., Proc. Phys. Soc. 78 (1961) 1206. 
[36 TwIGg H. E. and Frazer H. L. Microbeam Analysis, K. F. J. Heinrich Ed. (1982) 37.

[37] BÜCHNER A. R. and STIENEN J. P. M., Mikrochim. Acta. Suppl. 6 (1975) 227.

[38] PACKWOOD R. H. and BROWN J. D., X-Ray Spectrom. 10 (1981) 138.

[39] BASTIN G. F. VAN LOO F. J. J. and HeIJLIGERS H. J. M., X-Ray Spectrom. 13 (1984) 91.

[40] BASTIN G. F., HeIJligers H. J. M. and VAN LOO F. J. J., Scanning 6 (1984) 58.

[41] BASTin G. F., HeiJligers H. J. M. and VAN LOO F. J. J., Scanning 8 (1986) 45.

[42] REHBACH W. and KARDUCK P., in: Microbeam Analysis 1988, D. E. Newbury Ed. (San Francisco Press, San Fransisco, 1988) 285.

[43] KARDUCK P. and REHBACH W., in: Microbeam Analysis 1988, D. E. Newbury Ed. (San Francisco Press, San Francisco, 1988) 277.

[44] Love G., CoX M. G. C. and ScotT V. D., J. Phys. D 11 (1978) 23.

[45] AUGUST H.-J. and WERNISCH J., Scanning 12 (1990) 14.

[46] WITTRY D. B. Ph. D. Thesis, California Institute of Technology, Pasadena, California, 1957.

[47] CRISS J. in: Quantitative Electron Microanalysis, K. F. J. Heinrich Ed.,NBS Special Publ. 298, National Bureau of Standards, U. S. Dept. of Commerce, Washington, D. C. (1968) 53.

[48] Ugarte D., Castellano G., Trincavelli J., Del Giorgio M., Riveros J. A., X-Ray Spectrom. 16 (1987) 249.

[49] CRISS J. and BIRKS L. S. in: The Electron Microprobe, T. D. McKinley, K. F. J. Heinrich and T. D. Wittry Eds. (John Wiley \& Sons, New York, 1966) 217.

[50] SEwell D. A., LOVE G. and SCOTT V. D., J. Phys. D 18 (1985) 1245.

[51] BASTIN G. F. and HeiJligers H. J. M. Quantitative Electron Probe Microanalysis of Carbon in Binary Carbides. Report of the University of Technology Eindhoven, Laboratory for Physical Chemistry, PO Box 513, 5600 MB Eindhoven, ISBN 90-6819-004-0 CIP 1985. 